\title{
Involvement of B3GALNT2 overexpression in the cell growth of breast cancer
}

\author{
TAISUKE MATSUO ${ }^{1}$, MASATO KOMATSU ${ }^{1}$, TETSURO YOSHIMARU ${ }^{1}$, KAZUMA KIYOTANI ${ }^{1}$, \\ YASUO MIYOSHI $^{2}$, MITSUNORI SASA ${ }^{3}$ and TOYOMASA KATAGIRI ${ }^{1}$ \\ ${ }^{1}$ Division of Genome Medicine, Institute for Genome Research, The University of Tokushima; \\ ${ }^{2}$ Department of Surgery, Division of Breast and Endocrine Surgery, Hyogo College of Medicine, \\ Hyogo 663-8501; ${ }^{3}$ Department of Surgery, Tokushima Breast Care Clinic, Tokushima 770-0052, Japan
}

Received August 24, 2013; Accepted October 7, 2013

DOI: $10.3892 /$ ijo.2013.2187

\begin{abstract}
A number of glycosyltransferases have been identified and biologically characterized in cancer cells, yet their exact pathophysiological functions are largely unknown. Here, we report the critical role of $\beta 1,3-N$ acetylgalactosaminyltransferase II (B3GALNT2), which transfers $\mathrm{N}$-acetylgalactosamine (GalNAc) in a $\beta 1,3$ linkage to $\mathrm{N}$-acetylglucosamine, in the growth of breast cancer cells. Comprehensive transcriptomics, quantitative PCR and northern blot analyses indicated this molecule to be exclusively upregulated in the majority of breast cancers. Knockdown of B3GALNT2 expression by small interfering RNA attenuated cell growth and induced apoptosis in breast cancer cells. Overexpression of B3GALNT2 in HEK293T cells prompted secretion of the gene product into the culture medium, suggesting that B3GALNT2 is potentially a secreted protein. Furthermore, we demonstrated that B3GALNT2 is N-glycosylated on both Asn-116 and Asn-174 and that this modification is necessary for its secretion in breast cancer cells. Our findings suggest that this molecule represents a promising candidate for the development of a novel therapeutic targeting drug and a potential diagnostic tumor marker for patients with breast cancer, especially TNBC.
\end{abstract}

\section{Introduction}

Breast cancer is a highly heterogeneous disease that is currently classified by the expression profiling of estrogen receptor $(\mathrm{ER})$, progesterone receptor $(\mathrm{PgR})$ and the human epidermal growth factor receptor 2 (HER2) $(1,2)$. Endocrine

Correspondence to: Dr Toyomasa Katagiri, Division of Genome Medicine, Institute of Genome Research, The University of Tokushima, 3-18-15 Kuramoto-cho, Tokushima 770-8503, Japan

E-mail: tkatagi@genome.tokushima-u.ac.jp

Key words: glycosyltransferase, molecular target, breast cancer, secreted protein therapies such as tamoxifen and aromatase inhibitor have produced a significant improvement in outcomes for patients with ER-positive breast cancer. The HER2-targeting therapies, such as trastuzumab and lapatinib, have significantly improved the outlook for patients with HER2-positive breast cancer. However, increased risk of endometrial cancer with long-term tamoxifen administration and of bone fracture due to osteoporosis in postmenopausal women undergoing aromatase inhibitor treatment are recognized side effects (3-5). In addition, triple negative breast cancer (TNBC), defined as tumors that are characterized by lack of ER, PgR and HER2, accounts for $\sim 15 \%$ of all breast cancers and shows significantly poorer prognosis compared with other types of breast cancers because of a lack of clinically established targeted therapies $(6,7)$. Due to the emergence of these side effects, endocrine-resistant and chemo-resistant breast cancers and TNBC, it is necessary to search for novel molecular targets for drugs based on wellcharacterized mechanisms of action.

Current 'omics' technologies, including transcriptomics, are a very useful approach for identifying novel therapeutic targets for various cancers, including breast cancer (8-10). We previously used DNA microarray to analyze the genome-wide gene expression profiles of TNBCs and normal human vital organs including heart, lung, liver and kidney (10). After comparing the expression profiles of TNBCs and normal human tissues, we focused on $\beta 1,3-N$-acetylgalactosaminyltransferase II (B3GALNT2), which was significantly upregulated in TNBCs compared with normal breast ducts (10). B3GALNT2 was first identified as a novel glycosyltransferase having $\beta 1,3$-glycosyltranferase motifs, which are highly conserved in $\beta 1,3$-galactosyltranferase and $\beta 1,3-N$-acetylglucosaminyltranferase families, using a BLAST search (11). The purified putative catalytic domain of this protein reportedly has $\mathrm{N}$-acetylgalactosaminyltransferase in vitro activity and $\beta 1,3$-linkage as determined by NMR spectroscopic analysis. However, to date, no reports have characterized the biologic function of B3GALNT2 or the significance of its transactivation in clinical breast cancer cell growth.

Glycosylation plays crucial roles in a variety of biological functions, such as cell-cell and cell-substrate interactions, differentiation and signal transduction in tumor cells (12). The glycoproteins of tumor cells are often aberrant, both in structure and in quantity, leading to abnormal biological 
functions, including cell proliferation, migration, invasion and transformation (13-15). Although these alterations are caused by the dysregulated expression or structure of specific glycosyltransferase (16), their overview remains poorly understood.

In this study, we show that B3GALNT2 is overexpressed in breast cancers including TNBC and that downregulation of $B 3 G A L N T 2$ results in a significant reduction of breast cancer cell growth due to apoptosis induction. Moreover, we demonstrate that overexpression of B3GALNT2 results in its secretion into culture medium. Our findings suggest that B3GALNT2 represents a promising candidate for the development of molecular targeting therapy and might be a suitable diagnostic marker for breast cancer.

\section{Materials and methods}

Cell lines and specimens. Human breast cancer cell lines, BT-20, HCC1143, HCC1395, HCC1599, MCF-7, MDA-MB-453, OCOB-F, T47D and ZR-75-1 and human embryonic kidney fibroblast HEK293T cells were obtained from the American Type Culture Collection (ATCC, Rockville, MD, USA). BSY-1 cell line was a kind gift from Dr Takao Yamori of the Division of Molecular Pharmacology, Cancer Chemotherapy Center, Japanese Foundation for Cancer Research. All cells were cultured under conditions recommended by the ATCC as previously described (17). We monitored the cell morphology of these cell lines by microscopy and confirmed that they had maintained their morphologic states in comparison with the original morphologic images. No mycoplasma contamination was detected in the cultures of any of these cell lines using a Mycoplasma Detection kit (Takara, Kyoto, Japan) in 2011. A total of 30 TNBCs and 13 normal mammary tissues were obtained with informed consent from patients who were treated at the Tokushima Breast Care Clinic, Tokushima, Japan, as previously described (10). This study and the use of all clinical materials described above, was approved by the Ethics Committee of The University of Tokushima.

Reverse transcription and real-time PCR. Total RNA from clinical breast cancer samples and breast cancer cell lines was isolated using a NucleoSpin RNA II (Takara) according to the manufacturer's instructions. The poly A-RNA of normal human heart, liver, kidney, lung and mammary gland (MG) (Takata Clontech) was reverse transcribed as described previously $(17,18)$. Real-time PCR analysis was performed using Power SYBR Green PCR Master Mix (Life Technologies, Carlsbad, CA, USA) using an ABI PRISM 7500 Real-Time PCR system (Life Technologies) according to the manufacturer's instructions. Gene-specific primers used for real-time PCR were as follows: 5'-AAGACCTGTGAGACAGGAATGC-3' and 5'-GTTCTGGGTGAAAGTGCCAG-3' for B3GALNT2 and 5'-ATTGCCGACAGGATGCAG-3' and 5'-CTCAGGAGGA GCAATGATCTT-3' for $A C T B$ as a quantitative control.

Northern blot analysis. Isolation of mRNA from breast cancer cell lines was performed using mRNA Purification Kit 4 (GE Healthcare, Buckinghamshire, UK) according to the manufacturer's instructions. The northern blot for breast cancer cell lines was prepared as described previously (17). The breast cancer blot and human multiple tissue blots
(MTN and MTN II) were hybridized with $\left[\alpha^{32} \mathrm{P}\right]-\mathrm{dCTP}$ labeled PCR products of B3GALNT2 by RT-PCR using a primer set as follows: 5'-TGATGTGGTAGTTGGCGTGT-3' and 5'-AGAACTCCCCCTCCATCATT-3'. Prehybridization, hybridization and washing were performed as described previously (17). The blots were autoradiographed with intensifying screens at $-80^{\circ} \mathrm{C}$ for 14 days.

Gene silencing effect by siRNA treatment. BT-20, MDAMB-453 and ZR-75-1 cells were plated onto 12-well plates $\left(5 \times 10^{4}, 5 \times 10^{4}\right.$ and $2.5 \times 10^{4}$ cells/well, respectively). Transfection of $10 \mathrm{nM}$ siRNAs was performed using Lipofectamine RNAiMax (Life Technologies) according to the manufacturer's instructions. The target sequences for three B3GALNT2 and a control EGFP siRNAs were 5'-GUCAACGUGUGCUUGUG AA-3' for siB3GALNT2-1, 5'-CGAGCUUCAAUUUGUUGC U-3' for siB3GALNT2-2, 5'-CCGGAAAGUGGCAGGAG UU-3' for siB3GALNT2-3 and 5'-GCAGCACGACUUCUUC AAG-3' for siEGFP. To evaluate the knockdown effect of the siRNAs by quantitative RT-PCR, total RNA was extracted from the siRNA-transfected cells at 6 days after siRNA transfection. The gene-specific primers are described above. These experiments were performed in duplicate. To quantify cell viability, MTT assays were performed using a Cell-Counting Kit-8 according to the manufacturer's recommendations (Dojindo, Kumamoto, Japan). Absorbance at $450 \mathrm{~nm}$ was measured with the microplate reader Infinite 200 (Tecan, Männedorf, Switzerland). These experiments were performed in duplicate (MDA-MB-453 and ZR-75-1 cells) or triplicate (BT-20 cells).

Plasmids. To construct the B3GALNT2 expression vectors, the entire coding sequence of B3GALNT2 cDNA was amplified by RT-PCR using KOD plus DNA polymerase (Toyobo, Osaka, Japan) and cloned into the pCAGGSn3FC expression vector in frame with Flag-tag at the $\mathrm{COOH}$ terminus. The primer sets of B3GALNT2-wild-type (WT) was as follows: 5'-ATAAG AATGCGGCCGCATGCGAAACTGGCTGGTGC-3' and 5'-CCGCTCGAGTCTTGCTTGACATCGACAAGG-3' (the underlined letters indicate the NotI or XhoI sites, respectively). We also performed conventional two-step mutagenesis PCR to generate mutants in which N116A and N174A were substituted to alanines, as described previously (14). The primer sets were 5'-GTAAACTACTCGCCATCACAAATC-3' and 5'-GATTT GTGATGGCGAGTAGTTTAC-3' for N116A, 5'-GTTTCCA GAGGGCCATCACTGTC-3' and 5'-GACAGTGATGGCCC TCTGGAAAC-3' for N174A (the underlined letters indicate the mutation sites, respectively). The DNA sequences of all constructs were confirmed by DNA sequencing (ABI3500XL, Life Technologies).

Fluorescence activated cell-sorting (FACS) analysis. FACS analysis was performed as previously described (10). Briefly, the cells were collected at 2, 4 and 6 days after treatment of siRNAs against B3GALNT2 or EGFP as a control. The cells were fixed by $70 \%$ ethanol at room temperature for $30 \mathrm{~min}$ and then incubated at $37^{\circ} \mathrm{C}$ for $30 \mathrm{~min}$ with $1 \mathrm{mg} / \mathrm{ml}$ RNase A, followed by staining with $20 \mu \mathrm{g} / \mathrm{ml}$ propidium iodide at room temperature for $30 \mathrm{~min}$ in the dark. The DNA content of 10,000 cells was analyzed with a FACSCalibur flow cytometer and CellQuest software (BD Biosciences, Franklin Lakes, NJ, USA). 
Western blot analysis. Western blot analysis was performed as previously described (19). Briefly, the cells were lysed in lysis buffer [50 mM Tris- $\mathrm{HCl}(\mathrm{pH} 8.0), 150 \mathrm{mM} \mathrm{NaCl}$, $0.5 \%$ Nonidet P-40, $0.5 \%$ CHAPS] including $0.1 \%$ protease inhibitor cocktail III (Calbiochem, San Diego, CA, USA). The cell lysates were incubated on ice for $30 \mathrm{~min}$ and centrifuged at 15,000 rpm for $15 \mathrm{~min}$ to remove cell debris. Then, the proteins were mixed with SDS sample buffer $[25 \mathrm{mM}$ Tris- $\mathrm{HCl}$ (pH 6.8), 0.8\% SDS, 5\% glycerol] and boiled for 5 min. After SDS-PAGE, membranes blotted with proteins were incubated with anti-Flag M2 (Sigma-Aldrich, St. Louis, MO, USA, F3165) or anti- $\beta$-actin (AC-15, Sigma-Aldrich, A-5441) monoclonal antibodies diluted at 1:5,000 and PARP rabbit polyclonal antibody (Cell Signaling Technology, 9542) diluted at 1:500, respectively. Finally, the membrane was incubated with horseradish peroxidase (HRP)-conjugated secondary antibody for $1 \mathrm{~h}$ and protein bands were visualized by enhanced chemiluminescence detection reagents (ECL, GE Healthcare).

Immunocytochemical staining. Immunocytochemical staining was performed as previously described (10). To examine the subcellular localization of the B3GALNT2 protein, HEK293T cells were plated onto an 8-well glass slide (Thermo Fisher Scientific, Rochester, NY, USA) at a density of $1.0 \times 10^{4}$ cells/ well and transfected with $0.2 \mu \mathrm{g}$ each of expression plasmids using FuGENE 6 reagent (Promega, Madison, WI, USA) according to the manufacturer's recommendations. To detect exogenous B3GALNT2-Flag, anti-Flag M2 mouse antibody was used at 1:1,000 and Alexa 488-conjugated anti-mouse antibody. The Golgi apparatus were visualized by staining with anti-Golgi-58k mouse monoclonal antibody (SigmaAldrich) (20). Cell morphology was analyzed by Alexa Fluor 488 phalloidin (Molecular Probes, Eugene, OR, USA) diluted at 1:1,000.

Inhibition of $N$-glycosylation. HEK293T cells were plated onto 6 -well dishes at a density of $2.0 \times 10^{5}$ cells/well and transfected with $1 \mu \mathrm{g}$ each of expression plasmids using FuGENE 6 reagent according to the manufacturer's instructions. To validate the $\mathrm{N}$-glycosylation of B3GALNT2, the cells were cultured with $10 \mu \mathrm{g} / \mathrm{ml}$ tunicamycin (Sigma-Aldrich), an inhibitor against $\mathrm{N}$-glycosylation, for $24 \mathrm{~h}$, at $4 \mathrm{~h}$ after transfection. Cells were then lysed with SDS sample buffer.

Preparation of secreted B3GALNT2. To detect secreted B3GALNT2 protein, we transfected B3GALNT2-expressing plasmids to HEK293T cells as described above. The medium was changed to DMEM with $0.1 \%$ FBS at $48 \mathrm{~h}$ after transfection. Cells were cultured for a further $48 \mathrm{~h}$ and then supernatants were collected. After removing debris by centrifugation at $15,000 \mathrm{rpm}$ for $15 \mathrm{~min}$, we performed acetone precipitation at $-30^{\circ} \mathrm{C}$ overnight. Then, the pellets were resuspended with SDS sample buffer.

Statistical analysis. Statistical analysis was conducted using Student's t-test. A P-value of $<0.05$ was considered to be statistically significant. Box blot analysis was performed with StatView J 5.0 software using the microarray data of 13 normal ducts and 30 TNBCs [the Gene Expression Omnibus database accession no. GSE38959; (10)].

\section{Results}

B3GALNT2 is upregulated in breast cancer specimens and cell lines. To identify the novel therapeutic targets for TNBC therapy, we previously performed genome-wide gene expression profile analysis of TNBC and normal human tissues by DNA microarray analysis (10). In parallel with this approach, we attempted to search for the genes that encode proteins containing glycosyltransferase motifs, either based on reported information or according to prediction by the protein-motif program SMART because we previously succeeded in identifying and characterizing the cancerspecific glycosyltransferase GALNT6, that plays a critical role for mammary carcinogenesis, as a drug target $(14,15)$. Among the glycosyltransferase genes that are upregulated in expression profiles of breast cancers, we focused on the $B 3 G A L N T 2$ gene, which encodes a glycosyltransferase, as a drug target for breast cancer. Gene expression profiling analysis showed significant overexpression of B3GALNT2 in TNBC cases, compared with normal ductal cells (Fig. 1A). Using quantitative RT-PCR, we verified that B3GALNT2 was upregulated $>2$-fold in 5 of 10 TNBC samples compared with clinically normal breast tissues, whereas B3GALNT2 was hardly expressed in the heart, lung, liver, kidney and mammary gland (Fig. 1B). Subsequent northern blot analysis of 10 breast cancer cell lines detected an approximately $4.7-\mathrm{kb}$ $B 3 G A L N T 2$ transcript at a high level in 8 of the 10 breast cancer cell lines that were examined (Fig. 1C).

Furthermore, multiple tissue northern blot analysis revealed that a 4.7-kb transcript of B3GALNT2 was slightly expressed in heart, skeletal muscle, spleen, kidney, liver, small intestine, placenta, lung, prostate and ovary, while a $2.4-\mathrm{kb}$ transcript was exclusively expressed in testis (Fig. 1D). According to the National Center for Biotechnology Information (NCBI) database, two representative transcripts of 4,755 nucleotides (B3GALNT2-V1, GenBank accession no. NM_152490) and 2,022 nucleotides (B3GALNT2-V2, GenBank accession no. $\mathrm{BC} 029564)$ that share the same open reading frame encoding a 500-amino acid protein seemed to correspond to the two bands observed in multiple tissue northern blot analysis (Fig. 1E).

Effect of B3GALNT2 on cell growth. To assess whether $B 3 G A L N T 2$ is essential for the growth or survival of breast cancer cells, we transfected synthetic oligonucleotide siRNAs against B3GALNT2 into the TNBC cell line, which consisted of BT-20 cells in which B3GALNT2 was highly expressed. The mRNA levels of B3GALNT2 in the cells transfected with siB3-1,-2 or -3 were significantly downregulated in comparison with cells transfected with siEGFP as a control (Fig. 2A). We also observed a significant decrease in the number of viable cells measured by MTT assay (Fig. 2A). Similarly, silencing of B3GALNT2 expression by siB3-3 markedly decreased cell proliferation of the non-TNBC cell lines MDA-MB-453 and ZR-75-1, in which B3GALNT2 was highly expressed (Fig. 2B and C). 


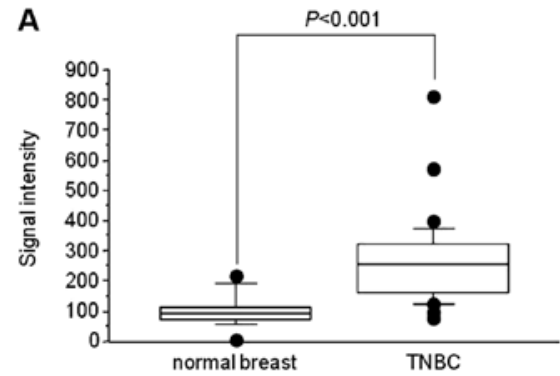

B

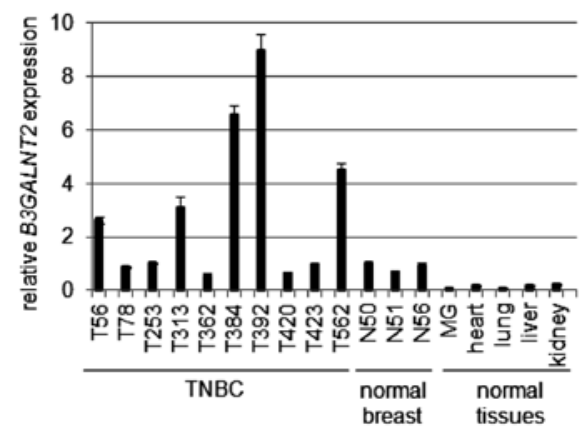

C

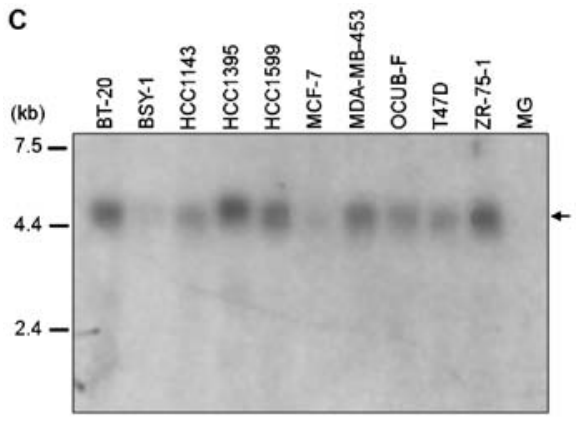

D
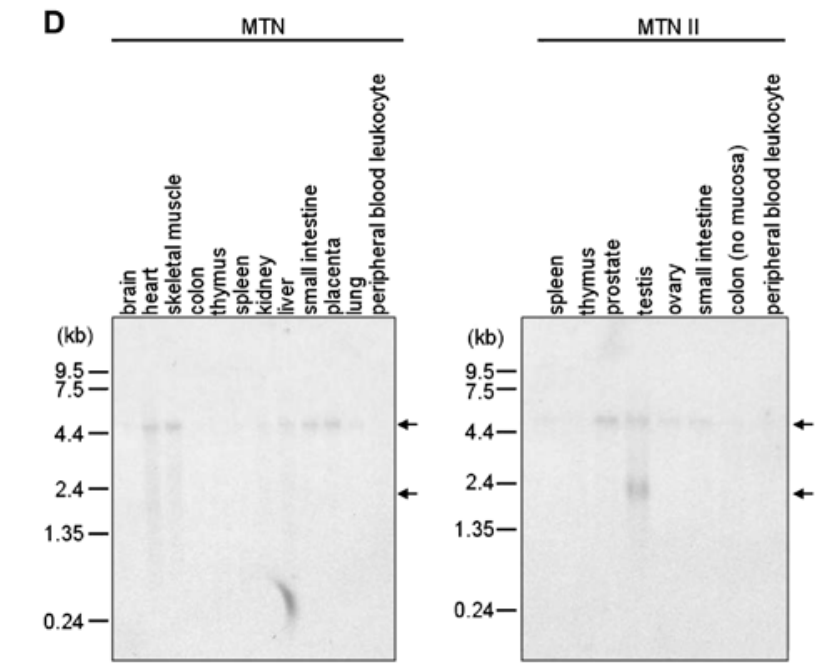

E
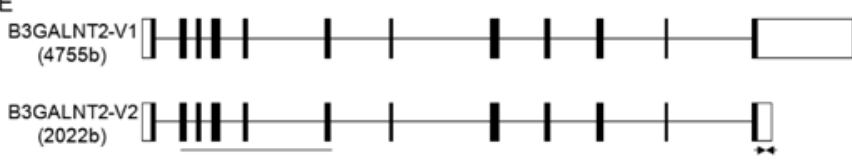

Figure 1. B3GALNT2 overexpression in breast cancer cells. (A) Box blot analysis of $B 3 G A L N T 2$ gene expression using microarray data from microdissected cells of 13 normal ductal tissues and 30 TNBC tissues. (B) Real-time RT-PCR result of B3GALNT2 in TNBC (T), normal breast ducts (N) and normal tissues $(\mathrm{n}=2)$. (C) Northern blot analysis of B3GALNT2 in 10 breast cancer cell lines and a mammary gland (MG). (D) Multiple human normal tissue northern blot analysis of B3GALNT2. (E) Genomic structure of $B 3 G A L N T 2$ variants. Black and white boxes indicate coding and non-coding regions, respectively. The arrows indicate a primer set for real-time RT-PCR. The line shows a probe position for northern blot analysis.
To further assess the knockdown effect of B3GALNT2, we performed FACS analysis and found that the percentage of sub-G1 population was clearly increased in a time-dependent manner in B3GALNT2-depleted BT-20 cells (Fig. 3A). In addition, we observed an obvious cleaved PARP at day 4 after treatment of siRNA against B3GALNT2 (siB3-3) (Fig. 3B). Interestingly, 4 days after transfection of siB3-3, severe disrupted cytoskeletal organization was observed by immunocytochemistry with fluorescence-labeled phalloidin (Fig. 3C), suggesting that depletion of B3GALNT2 resulted in suppression of breast cancer cell growth due to apoptosis. These findings suggest that B3GALNT2 is crucial for both TNBC and non-TNBC cell growth.

Secretory nature and $N$-glycosylation of B3GALNT2. The $B 3 G A L N T 2$ gene is reported to encode a type II transmembrane enzyme, which possesses a single transmembrane domain, a stem region and a $\mathrm{C}$-terminal catalytic domain for enzyme activity (21). To first investigate the subcellular localization of B3GALNT2 in mammalian cells, we transiently transfected the Flag-tagged B3GALNT2 construct (B3GALNT2-Flag) into HEK293T cells and then performed immunocytochemical staining analysis. B3GALNT2-Flag was observed to have highly intense staining in the Golgi apparatus, but it was also diffusely observed in cytoplasm (Fig. 4A). Furthermore, because many type II glycosyltransferases are found as secreted soluble enzymes through proteolytic cleavage of the stem region (22), we hypothesized that B3GALNT2 has a secretory nature. To investigate this possibility, HEK293T cells were transiently transfected with B3GALNT2-Flag and then western blot analysis with antiFlag antibody was performed using cell lysates and culture media. We detected a band of B3GALNT2-Flag in both cell lysates and culture media, but its molecular weight in culture media was smaller than that in cell lysate, suggesting the possibility that the B3GALNT2 protein was cleaved during its secretion into culture media (Fig. 4B).

$\mathrm{N}$-glycosylation on many glycosyltransferases is known to be associated with its biological functions, especially its secretion $(22,23)$. In addition, the NetNGlyc 1.0 server (http:// cbs.dtu.dk/services/NetNGlyc) and NCBI database predict that B3GALNT2 possesses two potential N-linked glycosylation at positions Asn-116 and Asn-174 that are completely conserved among other species, such as mouse, rat and Xenopus laevis (Fig. 4C). To confirm whether B3GALNT2 is $\mathrm{N}$-glycosylated, we treated HEK293T cells transfected with B3GALNT2-wild-type (B3GALNT2-WT) with tunicamycin, which is an N-glycosylation inhibitor and then analyzed the molecular weight of B3GALNT2-WT protein by western blot analysis. As expected, tunicamycin treatment resulted in a size reduction of B3GALNT2-WT (Fig. 4D, WT). Next, to assess the N-glycosylated amino acids of B3GALNT2, we constructed the expression plasmids of N116A or N174A single mutation and N116AN174A double mutation, in which the conserved asparagine residues at position 116 or 174 were replaced by alanine residues. When HEK293T cells were transfected with these plasmids without tunicamycin treatment, the molecular weight of N116A and N174A was smaller than that of B3GALNT2-WT, respectively and the molecular weight of N116AN174A double mutant was signifi- 
A

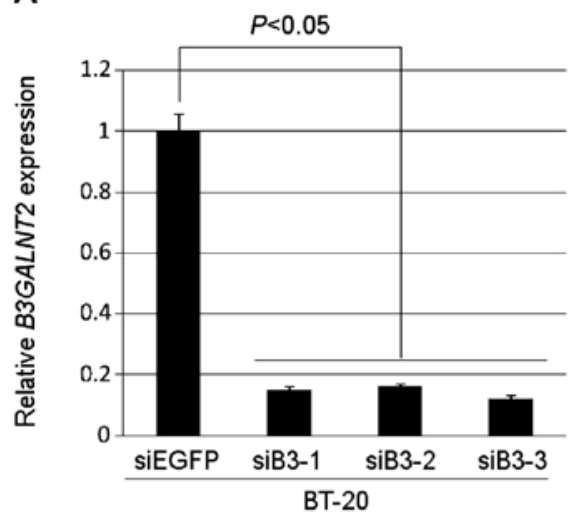

B

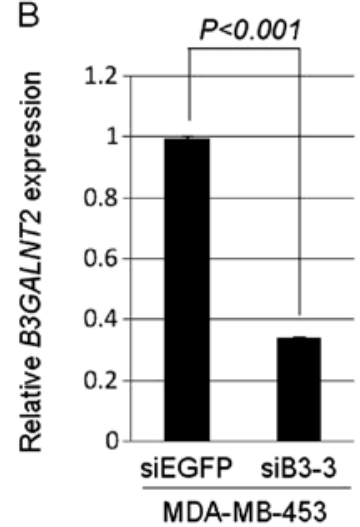

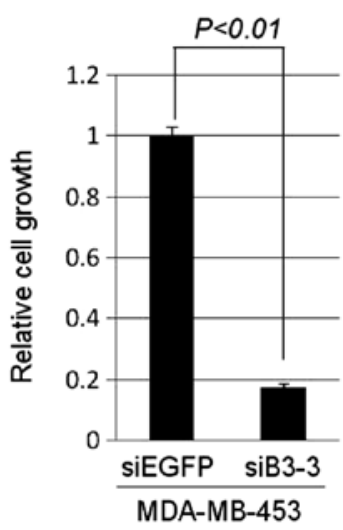

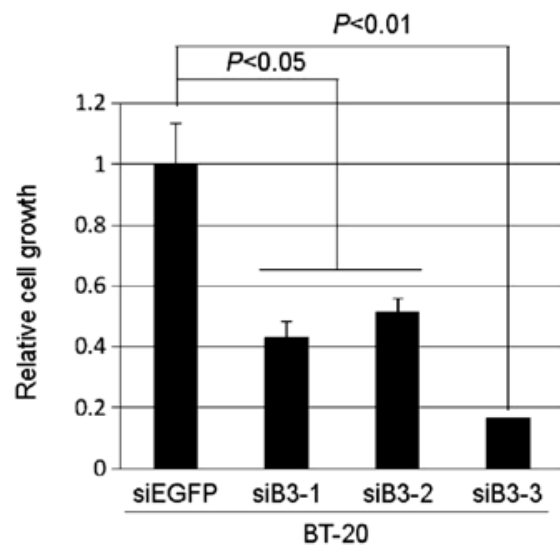

C

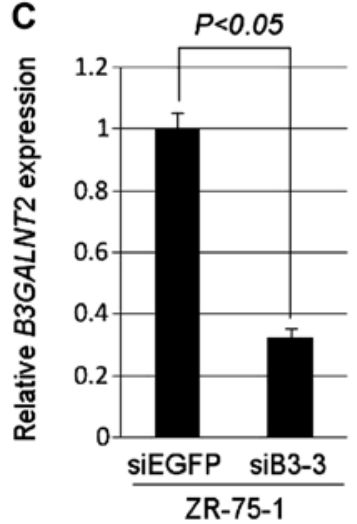

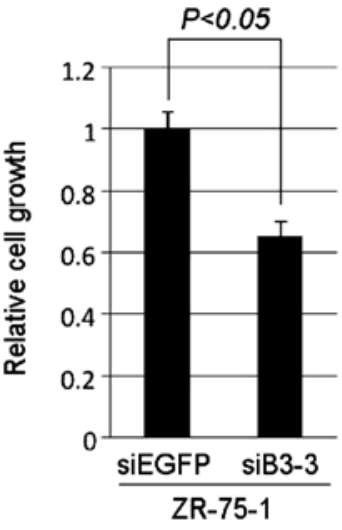

Figure 2. The knockdown effects of B3GALNT2 on cell proliferation by siRNA in BT-20 (A), MDA-MB-453 (B) and ZR-75-1 (C) cells. Real-time PCR of $B 3 G A L N T 2$ in siEGFP- or siB3GALNT2-treated cells $(\mathrm{n}=2)$. ACTB was used as a quantitative control for real-time RT-PCR. Cell proliferation was determined by MTT assay (BT-20; $\mathrm{n}=3$, MDA-MB-453 and ZR-75-1; $\mathrm{n}=2$ ).
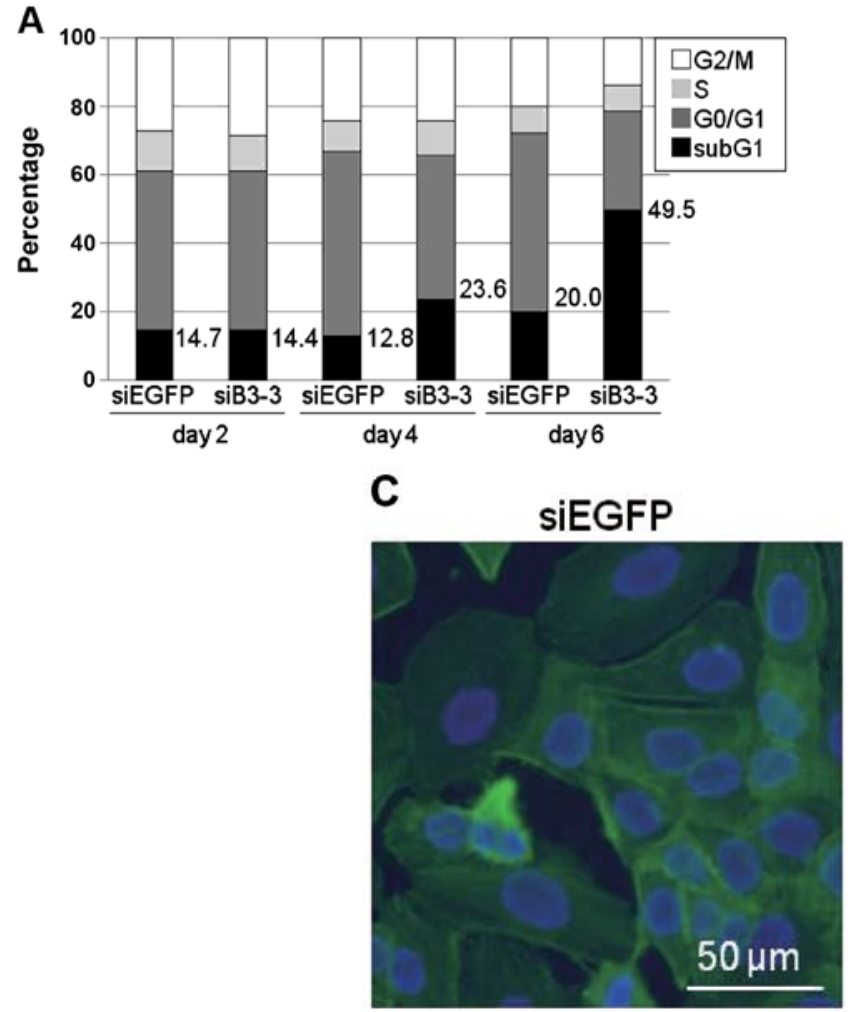

B

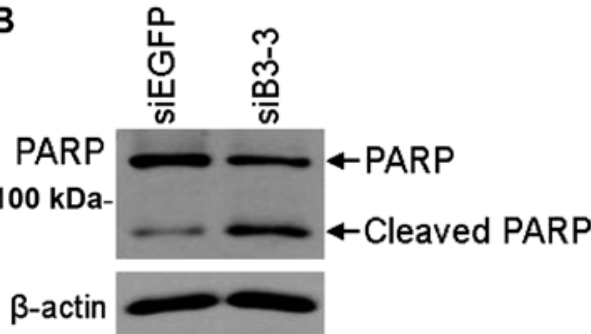

siB3-3

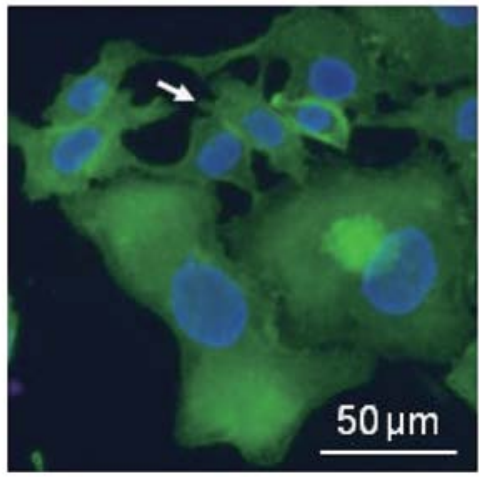

Figure 3. Knockdown of B3GALNT2-induced apoptosis in BT-20 TNBC cells. (A) FACS analysis of B3GALNT2-depleted BT-20 cells at each time-point. Numbers are the percentage of the sub-G1 population. (B) Cleaved PARP in BT-20 cells at 4 days after siRNA treatment was detected by western blot analysis. (C) Immunocytochemical staining in BT-20 cells at 4 days after siRNA treatment using Alexa 488-conjugated phalloidin (green) and DAPI (blue). The arrows indicate the altered cell morphology. 
A

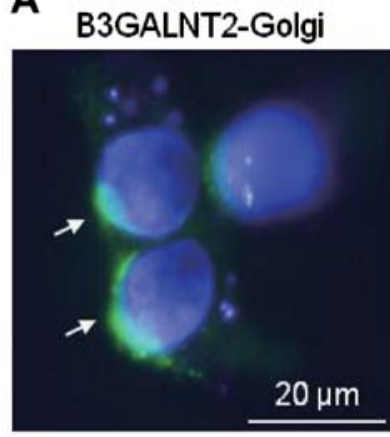

B

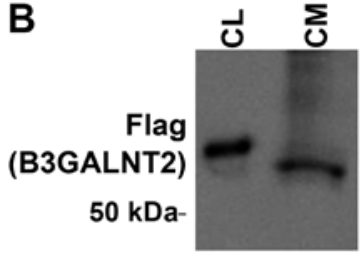

C

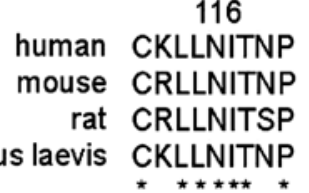

D (B3GALNT2) 50

\section{GFQRNITVK}

GFQRNITVK

GFQRNITVK

GFQRNITVK

\section{B3GALNT2-cytoplasm}

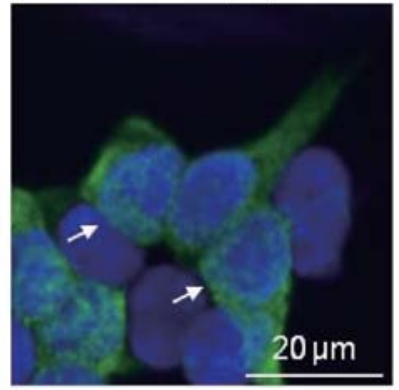

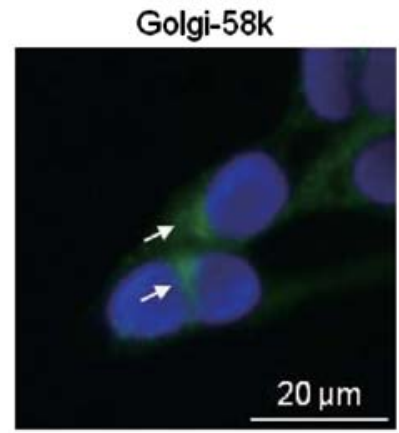

E

$\mathrm{CL}$

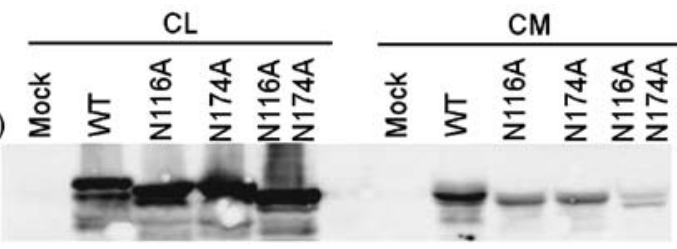

$\beta$-actin

Flag
(B3GALNT2)
$50-$

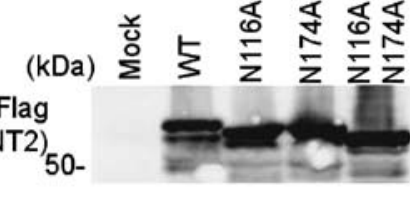
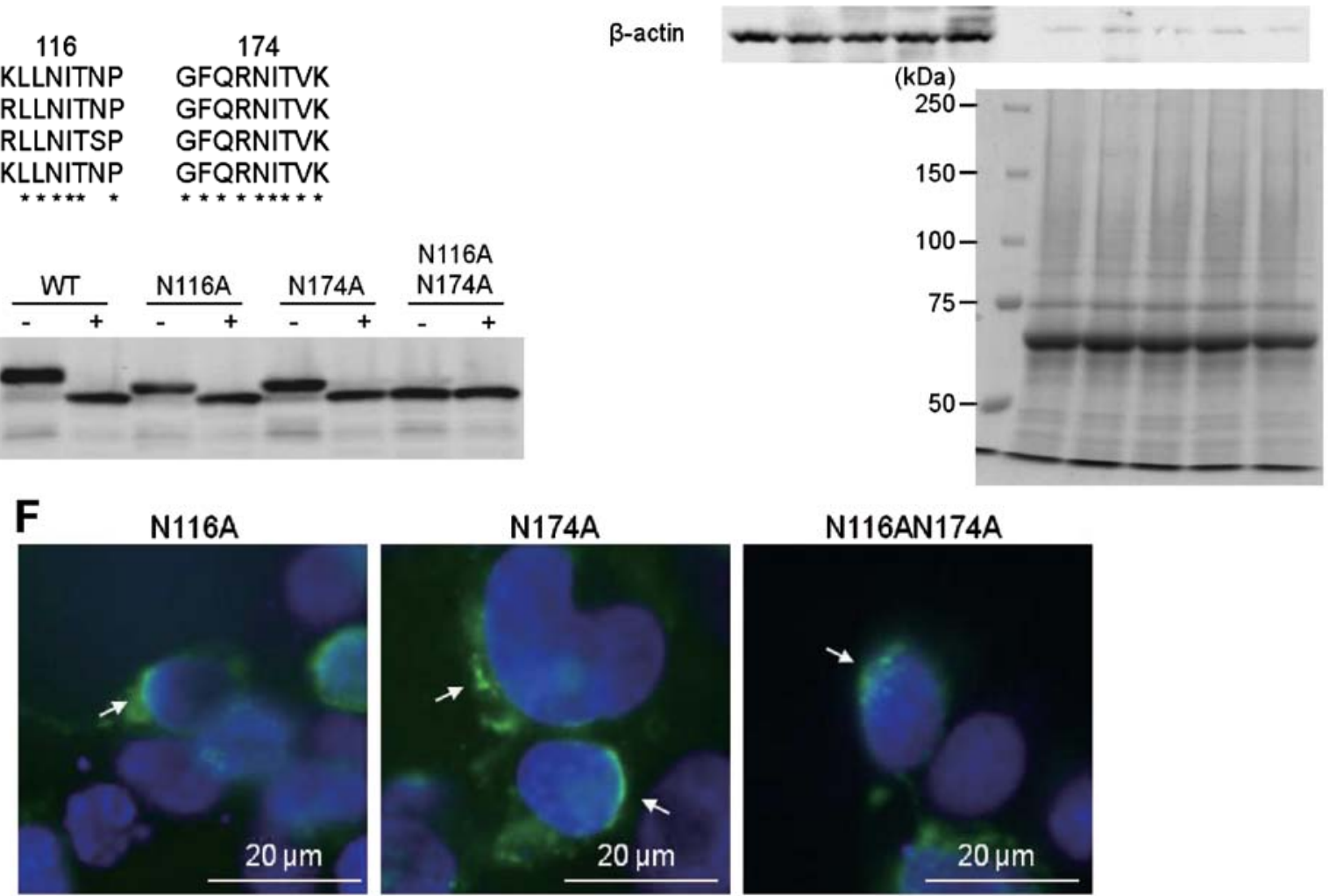

Figure 4. Subcellular localization, secretion and N-glycosylation of B3GALNT2 in B3GALNT2-overexpressing HEK293T cells. (A) Intracellular localization of B3GALNT2 was analyzed by immunocytochemical staining using anti-Flag antibody and Alexa 488-conjugated antibody (green). The Golgi apparatus were visualized by anti-Golgi-58k antibody (green). Nuclei were stained with DAPI (blue). The arrows indicate the localization of B3GALNT2-Flag or Golgi-58k. (B) B3GALNT2 of cell lysate (CL) and culture media (CM) from HEK293T cells, which were transiently expressing B3GALNT2-Flag, was detected by western blot analysis. (C) Homology search of two glycosylation sites of human B3GALNT2 with mouse (NP_848755), rat (NP_001138323) and Xenopus laevis (NP_001084830). Asterisks indicate the conserved amino acid residues among these species. (D) B3GALNT2-overexpressing HEK293T cells were cultured with (+) or without (-) tunicamycin (Tm). B3GALNT2-Flag was detected by western blot analysis using Flag monoclonal antibody. (E) B3GALNT2 of cell lysates (CL) and culture media (CM) was detected by western blot analysis. A comparison of the total protein amounts from culture media was performed by CBB staining. (F) Immunocytochemical staining was performed using anti-Flag antibody (green) and DAPI (blue). The arrows indicate the localization of B3GALNT2-Flag.

cantly smaller. In addition, tunicamycin treatment induced a decreased molecular mass of N116A and N174A, although the N116AN174A double mutant mass was not decreased (Fig. 4D).

To evaluate the effect of $\mathrm{N}$-glycosylation on the secretion of the B3GALNT2 protein, we examined its secretion in HEK293T cells transfected with B3GALNT2-WT, -N116A, -N174A and N116AN174A, respectively. The secretion of B3GALNT2N116A or -N174A was reduced compared with WT, while the secretion of N116AN174A mutant in culture media was drastically reduced (Fig. 4E). However, all of the mutants were mainly localized to the Golgi apparatus as well as B3GALNT2-WT 
(Fig. 4F), thus suggesting no effects of these mutants on its Golgi retention. Taken together, both Asn-116 and Asn-174 amino acids of B3GALNT2 are N-glycosylation sites and their $\mathrm{N}$-glycosylation is necessary for B3GALNT2 secretion.

\section{Discussion}

Glycosylation is a posttranslational modification and is associated with various physiologic events. The aberrant expression of glycosyltransferase and the immature glycan structure of proteins and lipids are observed in many cancers. These phenomena are also involved in the development and progression of cancers $(13-16,24)$. Abnormalities of the glycan structure of glycoproteins are frequently observed in breast cancer cells (13-15). In particular, we previously identified and characterized the oncogenic roles of a cancer-specific glycosyltransferase, UDP- $N$-acetyl- $\alpha$-D-galactosamine (GalNAc): polypeptide $N$-acetylgalactosaminyltransferase 6 (GALNT6) that regulated cell proliferation and cytoskeleton structure through aberrant $O$-glycosylation and stabilization of an oncoprotein mucin 1 (MUC1) (14) and fibronectin (15), which indicated that the development of GALNT6 inhibitors would be valuable for breast cancer therapy. To further elucidate the oncogenic role of aberrant glycosyltransferase expression, we attempted to identify cancerspecific glycosyltransferases that are exclusively upregulated in breast cancers through the analysis of comprehensive gene expression profiles of TNBC and normal human tissues. In this study, we focused on a breast cancer-specific glycosyltransferase, B3GALNT2 and showed its potential as a druggable target by showing its critical roles in breast cancer cell growth.

B3GALNT2 was indicated to be the member of the $\beta 1,3$-glycosyltransferase ( $\beta 3 \mathrm{GT}$ ) family by having three $\beta 3 \mathrm{GT}$ motifs and its function was shown by in vitro analyses to be a synthesis of GalNAc $\beta 1-3$ GlcNAc $\beta 1-R$ structure on both $\mathrm{N}$-glycans and O-glycans of proteins (11). However, the biological and biochemical functions of B3GALNT2 have not been clarified in mammalian cells, including human cancer cells, primarily because the GalNAc $\beta 1-3$ GlcNAc $\beta 1-R$ structure has been reported only in $\alpha$-dystroglycan in mammalian cells (25). Recently, mutations in the B3GALNT2 gene were identified in individuals with dystroglycanopathy by wholeexome and Sanger sequencing technologies, suggesting that $\alpha$-dystroglycan is the potential substrate of B3GALNT2 (26). In contrast, the expression of $\alpha$-dystroglycan has been reported to be frequently downregulated in breast cancers (27). Moreover, Stevens et al (26) showed that exogenous V5-tagged B3GALNT2 was mainly localized in the endoplasmic reticulum (ER) of $\mathrm{C} 2 \mathrm{C} 12$ myoblasts, which is not concordant with our results indicating that exogenous B3GALNT2-Flag was mainly localized in the Golgi apparatus (Fig. 4A). This discrepancy may be due to differences in the experimental procedures employed, including the type of cell lines or the different expression vector constructs. Indeed, both previous findings and our results showed only exogenous expression of B3GALNT2 in mammalian cells. Hence, further study is necessary to clarify the biological roles or exact subcellularlocalization of glycosylated $\alpha$-dystroglycan in endogenous B3GALNT2-overexpressing breast cancer cells.

We first identified B3GALNT2 to be upregulated in TNBCs, but demonstrated that the silencing of B3GALNT2 expression by siRNA resulted in significant suppression of the growth of non-TNBC and TNBC cell lines (Fig. 2). Nevertheless, the overexpression of B3GALNT2 into HEK293 or NIH3T3 cells could not enhance cell proliferation (data not shown), indicating that $B 3 G A L N T 2$ is indispensable for the survival of breast cancer cells, but B3GALNT2 alone may not be sufficient for transformation activity. Furthermore, B3GALNT2 was shown to be a secreted protein (Fig. 4), but addition of the conditioned media of the B3GALNT2-transfected HEK293T cells into the culture media of HEK293A cells could not enhance cell growth (data not shown). These results suggest that the intrinsic glycosyltransferase activity of B3GALNT2 might be critical for breast cancer cell growth. However, it has been reported that GnT-V secreted from WiDr colon cancer cells is directly involved in tumor angiogenesis in a glycosylation-independent manner, thus providing biological importance for the secretion of this glycosyltransferase (28). Therefore, further studies are needed to clarify the precise biological roles of the secreted form of B3GALNT2.

Furthermore, we demonstrated that N-glycosylation at Asn-116 or Asn-174 of B3GALNT2 is critical for its efficient secretion, but the effects of these posttranslational modifications on its biological functions, including enzyme activity, are unknown. Some studies have shown that $\mathrm{N}$-glycosylation on glycosyltransferases is required for their proper-folding and/or enzymatic activities (29-31). Therefore, further study is necessary to clarify the precise pathophysiological roles of B3GALNT2 in mammary carcinogenesis through identification and characterization of its specific substrates and to screen inhibitors targeting glycosyltransferase activity of B3GALNT2 for potential breast cancer therapeutic applications.

In conclusion, we demonstrated that overexpression of cancer-specific glycosyltransferase B3GALNT2 is critical for the growth or survival in breast cancers, including TNBC and ER-positive breast cancer. Our findings showed the usefulness of B3GALNT2 as a promising diagnostic and/or therapeutic target for breast cancer.

\section{Acknowledgements}

We thank Drs Kaoru Takegawa (Department of Bioscience and Biotechnology, Faculty of Agriculture, Kyushu University) and Tomoya Fukawa (The University of Tokushima) for helpful discussions and Ms. Hinako Koseki for technical assistance. This study was supported by a Grant-in-Aid for Young Scientists (B) (grant nos. 23790369 and 25860240) from MEXT.

\section{References}

1. Jemal A, Siegel R, Ward E, Hao Y, Xu J, Murray T and Thun MJ: Cancer Statistics, 2008. CA Cancer J Clin 58: 71-96, 2008.

2. Di Cosmo S and Baselga J: Management breast cancer with targeted agents: Importance of heterogeneity. Nat Rev Clin Oncol 7: 139-147, 2010.

3. Berry DA, Cronin KA, Plevritis SK, Fryback DG, Clarke L, Zelen M, Mandelblatt JS, Yakovlev AY, Habbema JD and Feuer EJ: Cancer Intervention and Surveillance Modeling Network (CISNET) Collaborators: Effect of screening and adjuvant therapy on mortality from breast cancer. N Engl J Med 353: 1784-1792, 2005.

4. Early Breast Cancer Trialists' Collaborative Group (EBCTCG): Effects of chemotherapy and hormonal therapy for early breast cancer on recurrence and 15-year survival: an overview of the randomised trials. Lancet 365: 1687-1717, 2005. 
5. Adamo V, Iorfida M, Montalto E, Festa V, Garipoli C, Scimone A, Zanghì $\mathrm{M}$ and Caristi $\mathrm{N}$ : Overview and new strategies in metastatic breast cancer (MBC) for treatment of tamoxifen-resistant patients. Ann Oncol 18: 53-57, 2007.

6. Foulkes WD, Smith IE and Reis-Filho JS: Triple-negative breast cancer. N Engl J Med 363: 1938-1948, 2010.

7. Liedtke C, Mazouni C, Hess KR, André F, Tordai A, Mejia JA, Symmans WF, Gonzalez-Angulo AM, Hennessy B, Green M, Cristofanilli M, Hortobagyi GN and Pusztai L: Response to neoadjuvant therapy and longterm survival in patients with triple-negative breast cancer. J Clin Oncol 26: 1275-1281, 2008.

8. Petricoin EF III, Hackett JL, Lesko LJ, Puri RK, Gutman SI, Chumakov K, Woodcock J, Feigal DW Jr, Zoon KC and Sistare FD: Medical applications of microarray technologies: a regulatory science perspective. Nat Genet 32: 474-479, 2002.

9. Nishidate T, Katagiri T, Lin ML, Mano Y, Miki Y, Kasumi F, Yoshimoto $M$, Tsunoda $T$, Hirata $K$ and Nakamura $Y$ : Genome-wide gene-expression profiles of breast-cancer cells purified with laser microbeam microdissection: identification of genes associated with progression and metastasis. Int J Oncol 25: 797-819, 2004

10. Komatsu M, Yoshimaru T, Matsuo T, Kiyotani K, Miyoshi Y, Tanahashi T, Rokutan K, Yamaguchi R, Saito A, Imoto S, Miyano S, Nakamura Y, Sasa M, Shimada M and Katagiri T: Molecular features of triple negative breast cancer cells by genome-wide gene expression profiling analysis. Int J Oncol 42: 478-506, 2013

11. Hiruma T, Togayachi A, Okamura K, Sato T, Kikuchi N, Kwon YD, Nakamura A, Fujimura K, Gotoh M, Tachibana K, Ishizuka Y, Noce T, Nakanishi H and Narimatsu H: A novel human beta1,3-N-acetylgalactosaminyltransferase that synthesizes a unique carbohydrate structure, GalNAcbeta1-3GlcNAc. J Biol Chem 279: 14087-14095, 2004.

12. Hart GW and Copeland RJ: Glycomics hits the big time. Cell 143: 672-676, 2010.

13. Fuster MM and Esko JD: The sweet and sour of cancer: glycans as novel therapeutic targets. Nat Rev Cancer 5: 526-542, 2005.

14. Park JH, Nishidate T, Kijima K, Ohashi T, Takegawa K, Fujikane T, Hirata K, Nakamura Y and Katagiri T: Critical roles of mucin 1 glycosylation by transactivated polypeptide $\mathrm{N}$-acetylgalactosaminyltransferase 6 in mammary carcinogenesis. Cancer Res 70: 2759-2769, 2010.

15. Park JH, Katagiri T, Chung S, Kijima K and Nakamura $Y$ : Polypeptide $\mathrm{N}$-acetylgalactosaminyltransferase 6 disrupts mammary acinar morphogenesis through O-glycosylation of fibronectin. Neoplasia 13: 320-326, 2011

16. Potapenko IO, Haakensen VD, Lüders T, Helland A, Bukholm I, Sørlie T, Kristensen VN, Ling jaerde OC and Børresen-Dale AL: Glycan gene expression signatures in normal and malignant breast tissue; possible role in diagnosis and progression. Mol Oncol 4: 98-118, 2010

17. Park JH, Lin ML, Nishidate T, Nakamura $Y$ and Katagiri T: PDZ-binding kinase/T-LAK cell-originated protein kinase, a putative cancer/testis antigen with an oncogenic activity in breast cancer. Cancer Res 66: 9186-9195, 2006.

18. Kim JW, Akiyama M, Park JH, Lin ML, Shimo A, Ueki T, Daigo Y, Tsunoda T, Nishidate T, Nakamura Y and Katagiri T: Activation of an estrogen/estrogen receptor signaling by BIG3 through its inhibitory effect on nuclear transport of PHB2/REA in breast cancer. Cancer Sci 100: 1468-1478, 2009.
19. Shimo A, Nishidate T, Ohta T, Fukuda M, Nakamura Y and Katagiri T: Elevated expression of protein regulator of cytokinesis 1 , involved in the growth of breast cancer cells. Cancer Sci 98: 174-181, 2007.

20. Nogueira E, Fidalgo M, Molnar A, Kyriakis J, Force T, Zalvide J and Pombo CM: SOK1 translocates from the Golgi to the nucleus upon chemical anoxia and induces apoptotic cell death. J Biol Chem 283: 16248-16258, 2008

21. Paulson JC and Colley KJ: Glycosyltransferases. Structure, localization, and control of cell type-specific glycosylation. J Biol Chem 264: 17615-17618, 1989.

22. El-Battari A, Prorok M, Angata K, Mathieu S, Zerfaoui M, Ong E, Suzuki M, Lombardo D and Fukuda M: Different glycosyltransferases are differentially processed for secretion, dimerization, and autoglycosylation. Glycobiology 13: 941-953, 2003.

23. Kato T, Suzuki M, Murata T and Park EY: The effects of $\mathrm{N}$-glycosylation sites and the N-terminal region on the biological function of beta1,3-N-acetylglucosaminyltransferase 2 and its secretion. Biochem Biophys Res Commun 329: 699-705, 2005.

24. Brockhausen I: Pathways of O-glycan biosynthesis in cancer cells. Biochim Biophys Acta 1473: 67-95, 1999.

25. Yoshida-Moriguchi T, Yu L, Stalnaker SH, Davis S, Kunz S, Madson M, Oldstone MB, Schachter H, Wells L and Campbell KP: $\mathrm{O}$-mannosyl phosphorylation of alpha-dystroglycan is required for laminin binding. Science 327: 88-92, 2010.

26. Stevens E, Carss KJ, Cirak S, Foley AR, Torelli S, Willer T, Tambunan DE, Yau S, Brodd L, Sewry CA, Feng L, Haliloglu G, Orhan D, Dobyns WB, Enns GM, Manning M, Krause A, Salih MA, Walsh CA, Hurles M, Campbell KP, Manzini MC; UK10K Consortium, Stemple D, Lin YY and Muntoni F: Mutations in B3GALNT2 cause congenital muscular dystrophy and hypoglycosylation of $\alpha$-dystroglycan. Am J Hum Genet 92: 354-365, 2013

27. Sgambato A,Migaldi M,Montanari M,Camerini A,Brancaccio A Rossi G, Cangiano R, Losasso C, Capelli G, Trentini GP and Cittadini A: Dystroglycan expression is frequently reduced in human breast and colon cancers and is associated with tumor progression. Am J Pathol 162: 849-860, 2003.

28. Saito T, Miyoshi E, Sasai K, Nakano N, Eguchi H, Honke $\mathrm{K}$ and Taniguchi $\mathrm{N}$ : A secreted type of $\beta 1,6-\mathrm{N}-$ acetylglucosaminyltransferase-V (GnT-V) induces tumor angiogenesis without mediation of glycosylation. A novel function of GnT-V distinct from the original glycosyltransferase activity. J Biol Chem 277: 17002-17008, 2002.

29. Toki D, Sarkar M, Yip B, Reck F, Joziasse D, Fukuda M, Schachter $\mathrm{H}$ and Brockhausen I: Expression of stable human $\mathrm{O}$-glycan core 2 beta-1,6-N-acetylglucosaminyltransferase in Sf9 insect cells. Biochem J 325: 63-69, 1997.

30. Barbier O, Girard C, Breton R, Bélanger A and Hum DW: $\mathrm{N}$-glycosylation and residue 96 are involved in the functional properties of UDP-glucuronosyltransferase enzymes. Biochemistry 39: 11540-11552, 2000.

31. Christensen LL, Bross P and Ørntoft TF: Glycosylation of the N-terminal potential N-glycosylation sites in the human alpha1,3fucosyltransferase V and -VI (hFucTV and -VI). Glycoconj J 17: $859-865,2000$ 